\title{
Onset voltage modeling of micromachined colloid thrusters
}

\author{
Renato Krpoun* and Herbert R. Shea ${ }^{\dagger}$ \\ School of Engineering, Ecole Polytechnique Fédérale de Lausanne, CH-1015 Lausanne, Switzerland
}

The onset voltage of a colloid thruster is an important parameter in the design of micromachined thrusters. Because dimensions in MEMS thrusters are in the range of several tens of micrometers and because extractor electrodes can be only $5 \mu \mathrm{m}$ from the capillary emitters, the aspect ratios for MEMS thrusters are much lower than the one of individual stainless steel or silica glass needles. Therefore the results obtained with established analytical models, that do not consider any parasitic structures, deviate significantly from experimental results on MEMS thrusters. This paper presents a new method to determine the onset voltage of micromachined colloid thrusters by combining electrostatic FEM analysis, including a model of the formation of the Taylor cone, with the equilibrium equation between electrostatic and surface tension stress. The correlation between onset voltage results obtained using the method presented in this paper and experiments are in very good agreement and validate the method for this type of microfabricated thruster.

\section{Nomenclature}

E $\quad$ Electric field, $\mathrm{V} / \mathrm{m}$

$B_{i, p}$ Bernstein basis function

C Parametric function, representing conic section

$\mathbf{P}_{\mathbf{i}} \quad$ Vectors defining conic section

$r_{i} \quad$ Principal radii of curvature, $\mathrm{m}$

$r_{a} \quad$ Radius of curvature at the apex of the forming Taylor cone, $\mathrm{m}$

$t \quad$ Variable parameter

$w \quad$ Variable parameter

$\gamma \quad$ Surface tension, $\mathrm{N} / \mathrm{m}$

$\delta_{i k} \quad$ Kronecker-delta

$\epsilon_{0} \quad$ Permittivity of free space, $\mathrm{F} / \mathrm{m}$

$\kappa \quad$ Proportionality factor, $1 / \mathrm{m}$

$\sigma_{E} \quad$ Electrostatic stress, $\mathrm{N} / \mathrm{m}^{2}$

$\sigma_{\gamma} \quad$ Surface tension stress, $\mathrm{N} / \mathrm{m}^{2}$

Subscript

$i, k, p$ Variable numbers

\section{Introduction}

$\mathrm{T}^{\mathrm{w}}$

HIS paper reports on an improved method to estimate the onset voltage of colloid thrusters with complex geometries. Existing models for the onset voltage of colloid thrusters assume the emitter is a needle or capillary with effectively infinite aspect ratio (i.e., that one can ignore the effect on the electric field of any surrounding structures). In general the shape of the liquid surface is approximated by a paraboloid or a hyperboloid surface ${ }^{1-3}$ and the extractor electrode is placed at a distance from the emitter that is at least an order of magnitude larger than the tip radius of the emitter capillary. Recent studies have focused on the

\footnotetext{
*PhD Candidate, Microsystems for Space Technologies Laboratory, renato.krpoun@epfl.ch, AIAA Student Member.

$\dagger$ Assistant Professor, Microsystems for Space Technologies Laboratory, herbert.shea@epfl.ch, AIAA Member.
} 
influence of interference effects on the onset voltage in an array of nozzles. ${ }^{4,5}$ These models do not include the effect of surface tension and require a calibration coefficient which needs to be found experimentally. To determine the onset voltage of micromachined thrusters manufactured in our lab, ${ }^{6}$ we have developed a method that combines analytical and finite-element modeling.

The ability to spray ion complexes (monomers, dimers, etc.) using ionic liquids as fuels ${ }^{7}$ and thus the possibility to obtain a large specific impulse with colloid thrusters has sparked the interest in creating arrays of emitters to increase thrust. Ionic liquids, also known as molten salts, are liquid at room temperature, conductive, exhibit no measurable vapor pressure and partially wet silicon surfaces. Micromachined arrays of capillaries with individual extractor electrodes allow obtaining high emitter densities and thus higher thrust, but as design parameters are interlinked they have to be carefully chosen if an optimum packing density is to be achieved. Key factors that must be considered for micromachined eletrospray arrays include: 1) the electric field screening of adjacent emitters (fig. 1), 2) the low aspect-ratios of MEMS structures and 3) the distances of less than $100 \mu \mathrm{m}$ between emitters and extractor electrodes.
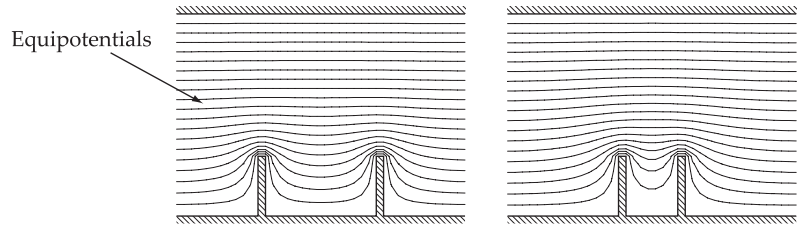

Figure 1. Schematic illustration of the effect of the electric field screening by adjacent capillaries.

The study was motivated by the observation of a large difference between the predicted voltage for spray onset obtained using the established analytical methods and our experimental results measured on microfabricated colloid thrusters consisting of arrays of silicon capillary emitters with integrated individual extractor electrodes. Therefore to predict more accurately the onset voltage a model was developed combining analytical methods with finite-element modeling to study such complex geometries and to investigate if a critical radius exists at the apex of the forming Taylor cone for which field self-amplification occurs.

\section{MEMS colloid thruster}

\section{A. Geometry}

A schematic cross section of the thruster geometry is shown in fig. 2 and features capillaries with $20 \mu \mathrm{m}$ diameter and $70 \mu \mathrm{m}$ height and extractor electrodes with 120 and $140 \mu \mathrm{m}$ diameter spaced as close as $5 \mu \mathrm{m}$ from the emitter tip. ${ }^{8}$ The SEM photograph in fig. 3a shows an array of emitters having a pitch of $250 \mu \mathrm{m}$. To simplify the geometry in the FEM analysis an axisymmetric geometry around the capillary is assumed. The adjecent capillaries are simulated by a solid structure placed at $250 \mu \mathrm{m}$ from the capillary.

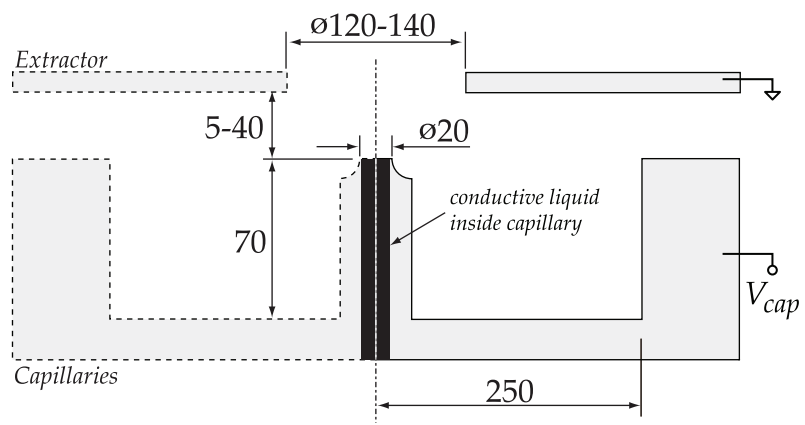

Figure 2. Geometry of the colloid thruster used for onset voltage simulation. The insulator between capillary emitters and extractor electrodes is not shown, dimensions are in microns. 


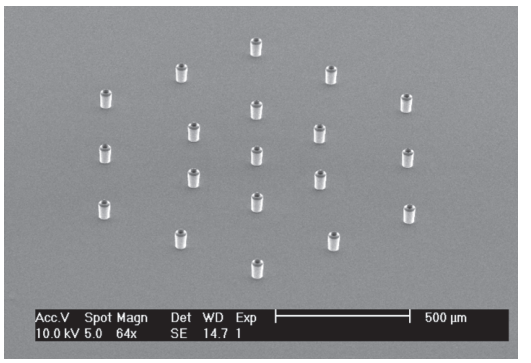

(a) Capillary Array

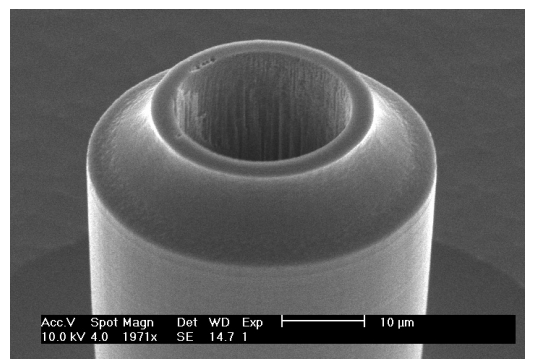

(b) Capillary Tip

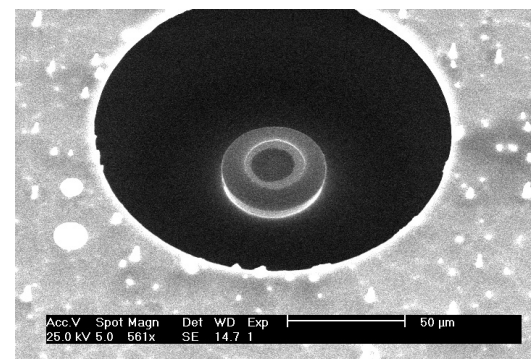

(c) Integrated Emitter

Figure 3. SEM photographs of the microfabricated colloid thruster. (a) array of silicon capillary nozzles, (b) detail of the capillary tip, (c) capillary seen through extractor electrode hole.

\section{B. Spray experiments}

Experiments were carried out in vacuum. ${ }^{9}$ The capillaries were mounted downwards and a voltage was applied to the capillaries while the extractor was held at ground. The emitted current was measured by means of a Faraday cup placed in the beam axis. The observed deviations in onset voltage can be caused by small variations in the surface tension of the fuel due to impurities and moisture. A second source of errors is the limited alignment tolerance between the extractor electrodes and the capillaries.

\section{Theoretical considerations}

The developed method avoids the difficulty of modeling hydrodynamic effects by assuming a purely static case. It can be best understood as a sequence of snapshots taken of the forming cone at the tip of a capillary or needle. As the voltage difference between extractor electrode and emitter increases the liquid at the tip of the capillary starts to deform, shown in figure 4 . For non-forced flow observations indicate that the liquid surface is bound within a cone. For the model a conic function has been selected that approximately reproduces the observed behavior and has the radius of curvature of the apex of the forming cone as parameter (see fig. 5). The method computes the equilibrium voltage between surface tension and electric field for various apex radii. From this data the onset voltage can be determined.
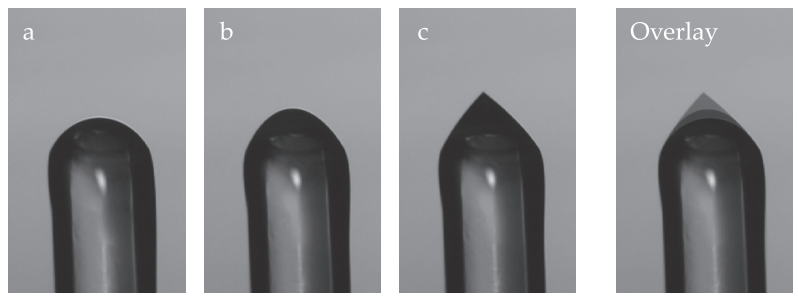

Figure 4. Sharpening of the liquid surface at the tip of a $360 \mu m$ outer diameter capillary. Photographs were taken at ambient pressure while spraying ionic liquid EMI-BF 4 , with a surface tension of $0.045 \mathrm{~N} / \mathrm{m}$. The extractor electrode (not visible in these images) is a plate placed a few millimeters above the capillary.

\section{A. Equilibrium between surface tension and electric stress}

When an electric field is applied between a capillary filled with a conductive liquid and an extractor electrode the liquid deforms into a shape where surface tension, $\sigma_{\gamma}$, balances the electric stress, $\sigma_{E}$. This equilibrium can be expressed as ${ }^{1}$

$$
\sigma_{E}=\sigma_{\gamma}
$$

The surface tension at the apex can be calculated using the Young-Laplace equation

$$
\sigma_{\gamma}=\gamma\left(\frac{1}{r_{1}}+\frac{1}{r_{2}}\right)
$$


where $r_{1}$ and $r_{2}$ are the principal radii of curvature of the meniscus and $\gamma$ is the surface tension of the liquid. For a symmetrical meniscus with radius $r_{a}$, as is the case at the apex of the cone the principal radii of curvature are idendentical and equation 2 simplifies into

$$
\sigma_{\gamma}=\frac{2 \gamma}{r_{a}}
$$

The electrical stress on the surface of the conducting liquid is given by the Maxwell stress tensor: ${ }^{10}$

$$
\sigma_{i k}=-\epsilon_{0}\left(\frac{1}{2} E^{2} \delta_{i k}-E_{i} E_{k}\right)
$$

where $E$ is the electrical field and $\epsilon_{0}$ the permittivity of free space. On the surface of the liquid the field $E$ has no tangential component therefore equation 4 becomes

$$
\sigma_{E}=\frac{1}{2} \epsilon_{0} E^{2} .
$$

Ignoring space charge effects a linear relation between electric field and voltage can be established. By replacing equations 3 and 5 into 1 and expressing the linear relation between electric field and voltage through a scaling factor, $\kappa$, the equilibrium equation becomes

$$
V=\frac{1}{\kappa} \sqrt{\frac{4 \gamma}{\epsilon_{0} r}} .
$$

\section{B. Modeling of the liquid surface at the tip of the capillary}

We chose to model the sharpening of the apex by a conic section, which corresponds to a straight line for an infinite radius of curvature, $r_{a}=\infty$, and a perfect cone for zero tip radius, $r_{a}=0$. An arc of a conic having such properties can be expressed mathematically as ${ }^{11}$

$$
\mathbf{C}(t)=\frac{B_{0,2}(t) \mathbf{P}_{\mathbf{0}}+B_{1,2}(t) w \mathbf{P}_{\mathbf{1}}+B_{2,2}(t) \mathbf{P}_{\mathbf{2}}}{B_{0,2}(t)+B_{1,2}(t) w+B_{2,2}(t)}
$$

where $B_{i, p}(t)$ are $p$ th degree Bernstein basis functions defined by

$$
\begin{aligned}
& B_{i, p}(t)=\left(\begin{array}{c}
p \\
i
\end{array}\right) t^{i}(1-t)^{n-i} \quad(i=0,1, \cdots, n) \\
& \left(\begin{array}{c}
p \\
i
\end{array}\right): \text { Binomial coefficient. }
\end{aligned}
$$

Equation 7 defines a one parameter family of conics passing through $\mathbf{P}_{\mathbf{0}}$ and $\mathbf{P}_{\mathbf{2}}$. The conic is further tangent to the chords $\left[\mathbf{P}_{\mathbf{0}}, \mathbf{P}_{\mathbf{1}}\right]$ and $\left[\mathbf{P}_{\mathbf{1}}, \mathbf{P}_{\mathbf{2}}\right]$. Fig. 5 plots the function, bound by the Taylor angle asymptotes for different apex radii. The function is spun around its axis of symmetry. For each $w$ a radius of curvature, $r_{a}$, at the tip of the apex can be associated

$$
w \longrightarrow r_{a}=r_{a}(w)
$$

\section{Onset voltage determination - Methodology}

The simulation method creates an axisymmetric surface representing the forming Taylor cone by spinning the function 7 around its axis of symmetry. For various apex radii a finite-element model is then constructed by placing the generated surface on top of the static geometry of figure 2. A voltage is applied on the capillaries and the extractor electrodes are held at ground. The electric field is then computed using the software tool ANSYS. To limit the number of elements and decrease computation time only a small slice of the axisymmetric geometry is modeled, as shown in fig. 6 . To simulate structures varying by several orders of magnitude, as is required by the decreasing tip radius, the chosen FEM elements are variable in size.

Once meshing is completed the electric field for a predetermined potential is computed and the linearity factor between electric field and voltage, $\kappa$, determined. For each specific apex radius the equilibrium voltage, i.e. the voltage at which the equilibrium between surface tension force and electrostatic forces are equal (eq. 6), is then determined and the results are plotted. 


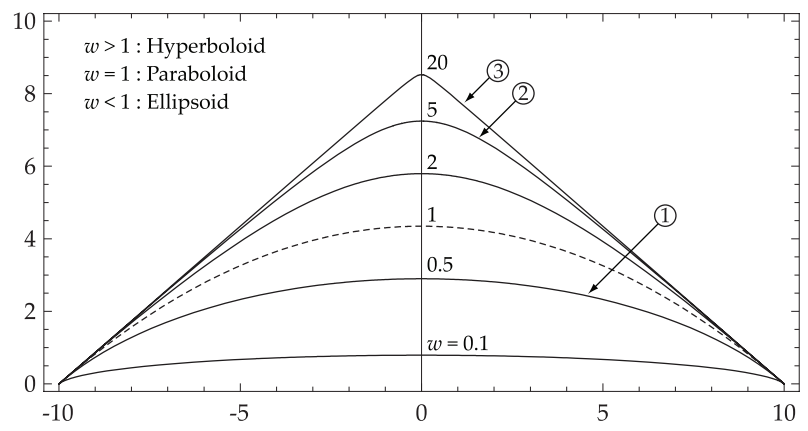

Figure 5. Cross-section of the conic function used to model the formation of a Taylor cone.

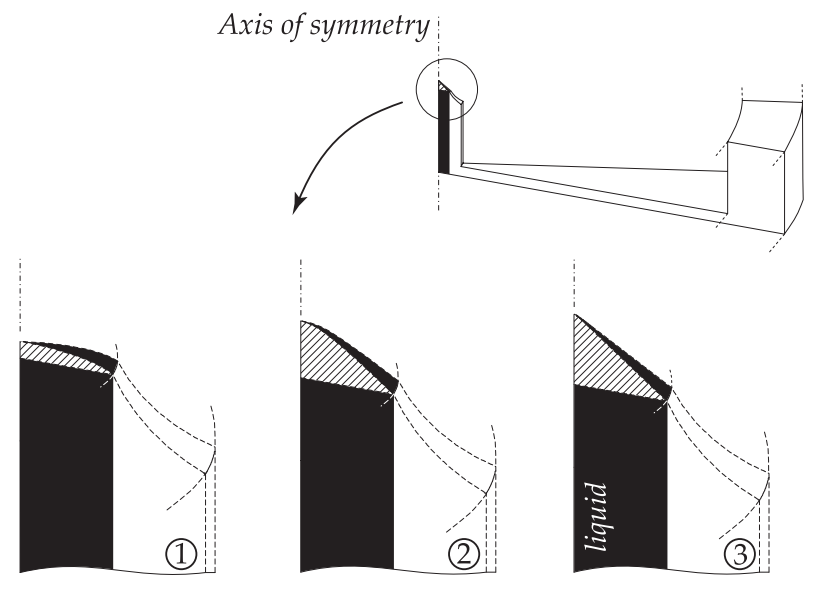

Evolving Taylor cone at tip of capillary (shaded)

Figure 6. Slice of the geometry used to compute the electric field distribution for three radii of curvature. The liquid is assumed to be a perfect conductor.

\section{Results}

\section{Correlation with experimental results}

The computed values for the geometry described above with an extractor-emitter spacing of $40 \mu \mathrm{m}$ and $120 \mu \mathrm{m}$ extractor diameter is shown in fig. 7, plotting the equilibrium voltage as a function of radius of curvature of the apex of the liquid. From right to left: as the voltage increases the radius at the apex of the forming cone decreases. For the capillary geometries we studied, at large tip radii, there is first a region where the electric field is focused at sharp edges in the static geometry of the thruster. As the voltage is increased and the liquid shape becomes more cone-like, the electric field then is focused on the apex of the cone. Finally there is a region of constant voltage, the critical voltage, where the equilibrium voltage no longer depends on apex radius of curvature.

Spraying is assumed to start at electric fields in the order of $1 \mathrm{~V} / \mathrm{nm}$, indicated by a dashed line in the graph. Turn-on voltage is thus the voltage where the $1 \mathrm{~V} / \mathrm{nm}$ line intersects the equilibrium voltage plot. The key observation is that, because of the flat region where the equilibrium voltage is independent of tip radius, the onset voltage can be determined very precisely with only an order of magnitude knowledge of electric field required for spraying.

This zone of constant equilibrium voltage can explain why existing models calculating the electric field at the tip of a needle with radii of several micrometers produce acceptable results. Although their tip radii are at least one order of magnitude above the necessary radius to obtain a sufficiently large electric field for spraying to occur they focus the field sufficiently that once the equilibrium voltage is reached the field self 


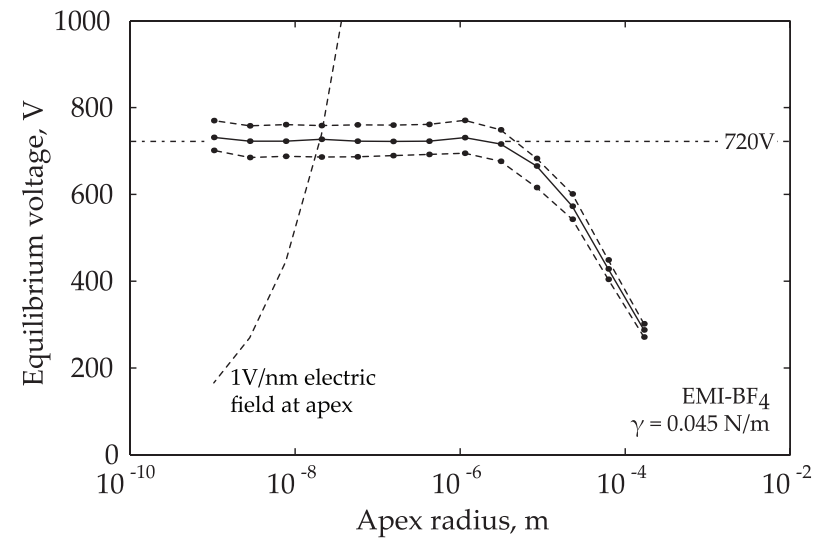

(a) Geometric sensitivity

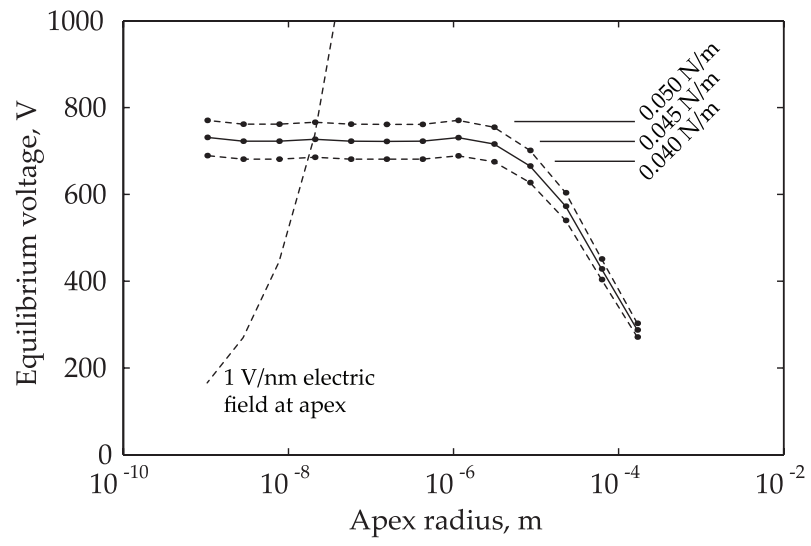

(b) Surface tension sensitivity

Figure 7. Plot of equilibrium voltage vs. apex radius for a thruster with an extractor electrode spaced at $40 \mu \mathrm{m}$ from the capillary tip and with an extractor diameter of $120 \mu \mathrm{m}$. The dashed lines above and below the curve show the sensitivity of the method for (a) the worst case if a geometric variation of all parameters by $5 \mu \mathrm{m}$ occurs and (b) of a change in surface tension.

amplifies and the Taylor cone forms. The two dashed curves in fig. 7a above and below the main plot indicate the sensitivity of the analysis with respect to geometrical variations. For both the capillary height, extractoremitter distance and extractor diameter have each been varied by $5 \mu \mathrm{m}$ to create a maximum increase or decrease of the equilibrium voltage. A comparison between experimental, simulation and analytical results is shown in table 1. As the extractor-emitter spacing is reduced, the onset voltage obtained using the analytical model deviates significantly from the experimental ones, whereas the voltage obtained from the method presented in this paper fit the measured value very well. In addition to geometric deviations the surface tension plays an important role, as shown in fig. 7b. For ionic liquids this observation is of particular importance as they readily absorb moisture when exposed to ambient environment. For the liquid EMI-BF 4 the surface tension measurement taken by Martino et $a l^{12}$ in a 'dry' environment resulted in a value which is $10 \%$ below the one obtained in a laboratory environment, which corresponds to a variation of more than $50 \mathrm{~V}$ in our simulation.

Table 1. Comparison between experimental, simulation and analytical results. The simulation results have been obtained with the method presented in this paper, while the analytical ones using the hyperboloid surface algorithm

\begin{tabular}{|c|c|c|c|c|}
\hline \multirow{2}{*}{$\begin{array}{c}\text { Emitter-extractor } \\
\text { distance }^{\mathrm{a}}(\mu \mathrm{m})\end{array}$} & \multirow{2}{*}{$\begin{array}{c}\text { Extractor } \\
\text { diameter }(\mu \mathrm{m})\end{array}$} & \multicolumn{3}{|c|}{ Onset Voltage (V) } \\
\hline & & Experimental $^{\mathrm{b}}$ & Simulation $^{\mathrm{c}}$ & Analytical $^{\mathrm{d}}$ \\
\hline 5 & 90 & $550-570$ & 530 & 155 \\
\hline 25 & 140 & $670-690$ & 700 & 515 \\
\hline 40 & 120 & 700 & 720 & 620 \\
\hline 40 & 140 & $700-720$ & 755 & 620 \\
\hline \multicolumn{5}{|c|}{$\begin{array}{l}{ }^{a} \text { Capillary height } 70 \mu \mathrm{m} \text {, capillary diameter } 20 \mu \mathrm{m} \text {. } \\
{ }^{\mathrm{b}} \text { Range of measured onset voltages at room temperature with EMI- } \mathrm{BF}_{4} \text {. } \\
{ }^{\mathrm{c}} \text { A surface tension of } 0.045 \mathrm{~N} / \mathrm{m} \text { was used for the simulation, following the methodology } \\
\text { described in this article. }\end{array}$} \\
\hline
\end{tabular}

\section{Influence of design parameter variations}

The method also allows to evaluate the impact of different parameters on a thruster design. Figure 8a shows the effect on the onset voltage of the distance between extractor and emitter. As expected a linear relationship can be seen as the extractor is moved away. An interesting result is shown in figure 8b where the 
bounding sidewalls are moved closer to the capillary emitter. An increase in onset voltage is observed because the parasitic structure starts interfering with the emitter and one should note that the curve corresponds well with experimental results reported in literature. ${ }^{4,5}$ Figures $8 \mathrm{c}$ and $8 \mathrm{~d}$ finally show the influence of the sidewalls on the onset voltage as a function of extractor electrode distance and of standoff height of the capillaries. These results also allow to find a compromise between minimum capillary pitch and crosstalk.

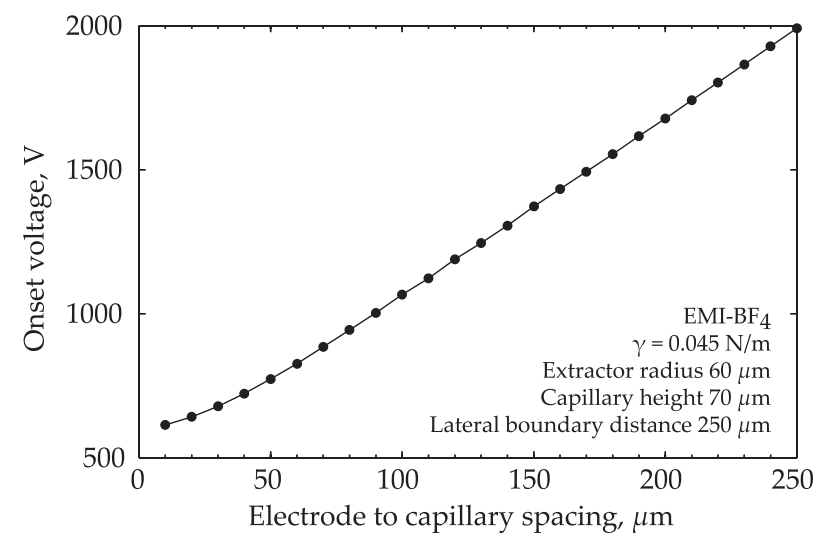

(a)

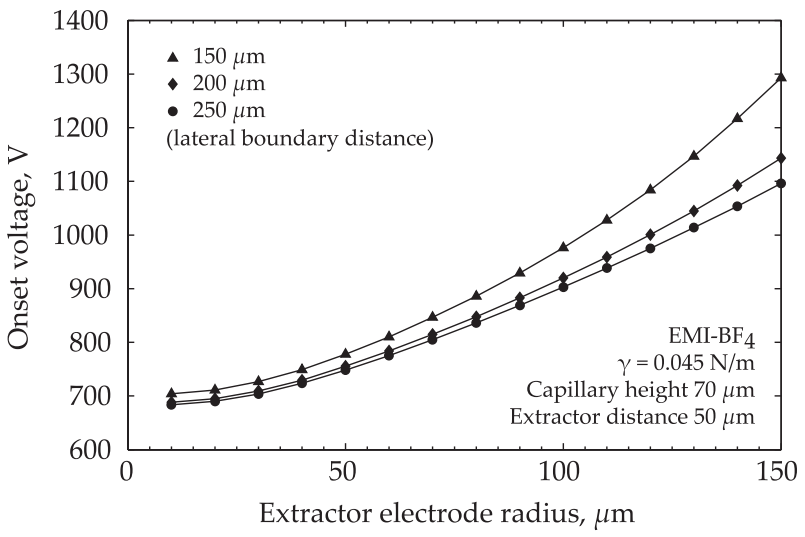

(c)

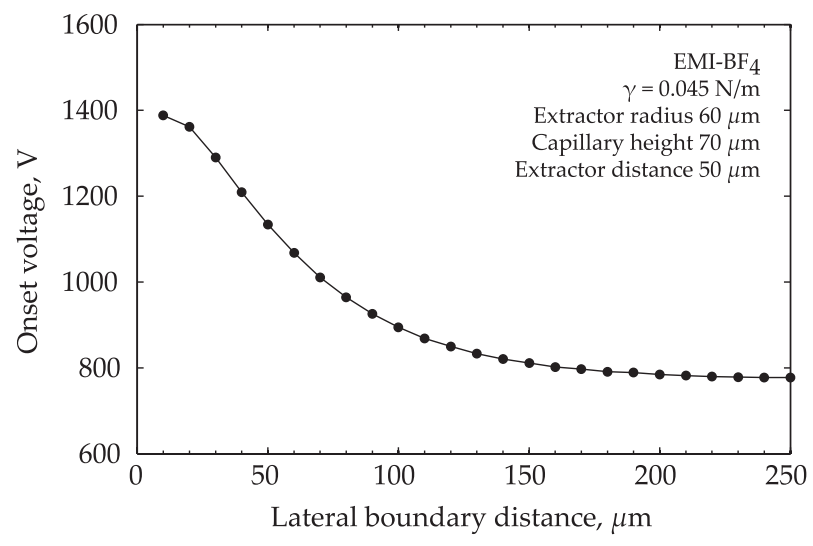

(b)

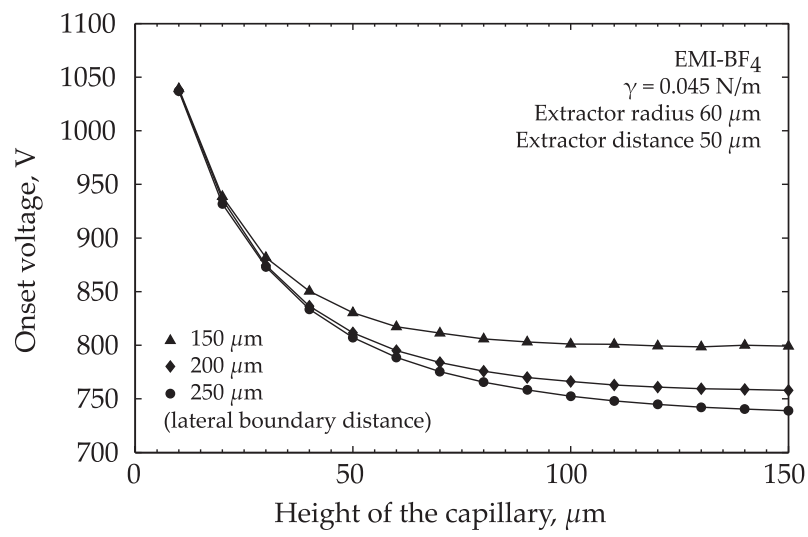

(d)

Figure 8. Plot of the onset voltage for a variation in (a) capillary emitter - extractor electrode distance, (b) distance between capillary and lateral sidewall, (c) extractor radius, and (d) height of the capillary emitter.

\section{Conclusion}

The problem of onset voltage determination in a colloid microthruster has been studied. The numerical method developed here describes the variation of the voltage needed to balance electric stress and surface tension at the apex of an an idealized liquid surface deforming into a Taylor cone. The results presented in this paper show that once a threshold apex radius has been reached the equilibrium voltage remains constant. For apex radii larger than this threshold value the electric field is weakened due to distortion by the surrounding structure, while for smaller radii the electric field is focused on the apex of the forming cone. We applied our method to one particular thruster configuration the subsequent future work will focus on other geometries and liquids to broaden the models range of validity. The model agrees with earlier analytical models that assume a sharp needle with infinite aspect ratio without considering the Taylor cone, but delivers a possible explanation why these models yield acceptable results for these types of geometries. 


\section{Acknowledgments}

The authors acknowledge the support from J. Gonzalez del Amo of the European Space Agency and thank Prof. J. P. W. Stark and Dr. K. Smith from Queen Mary, University of London, for their valuable advice.

\section{References}

${ }^{1}$ Prewett, P. and Mair, G., Focused ion beams from liquid metal ion sources, Electronic and electrical engineering research studies - Microengineering series, Research Studies Press LTD., 1991.

${ }^{2}$ Xiong, J., Sun, D., Zhou, Z., and Zhang, W., "Investigation of the onset voltage for the design of a microfabricated colloid thruster," IEEE/ASME Transactions on Mechatronics, Vol. 11, No. 1, 2006, pp. 66-74.

${ }^{3}$ Martínez-Sánchez, M., "16.522 Space Propulsion," MIT OpenCourseWare, URL: http://ocw.mit.edu/ [cited 31 October 2007], 2004.

${ }^{4}$ Regele, J., Papac, M., Rickard, M., and Dunn-Rankin, D., "Effects of capillary spacing on EHD spraying from an array of cone jets," Journal of Aerosol Science, Vol. 33, No. 11, 2002, pp. 1471-1479.

${ }^{5}$ Quang Tran Si, B., Byun, D., and Lee, S., "Experimental and theoretical study of a cone-jet for an electrospray microthruster considering the interference effect in an array of nozzles," Journal of Aerosol Science, Vol. 38, No. 9, 2007, pp. 924934 .

${ }^{6}$ Krpoun, R., Räber, M., Smith, K. L., Stark, J. P. W., and Shea, H. R., "Design and fabrication of an integrated MEMSbased colloid micropropulsion system," 30th International Electric Propulsion Conference, Florence, Italy, No. 099, September 17-20 2007.

${ }^{7}$ Romero-Sanz, I., Bocanegra, R., and Fernandez de la Mora, J., "Source of heavy molecular ions based on Taylor cones of ionic liquids operating in the pure ion evaporation regime," J. Appl. Phys., Vol. 94, No. 5, September 2003, pp. $3599-3605$.

${ }^{8}$ Krpoun, R., Räber, M., and Shea, H. R., "Microfabrication and test of an integrated colloid thruster," 21st IEEE International Conference on Micro Electro Mechanical Systems, No. 0234, IEEE, 2008, (accepted for presentation).

${ }^{9}$ Krpoun, R. and Shea, H. R., "Micromachined electric propulsion using ionic liquids as fuel," 14th International Workshop on the Physics of Semiconductor Devices, Mumbai, 2007.

${ }^{10}$ Landau, L. D., Lifshitz, E. M., and Pitaevskii, L. P., Electrodynamics of Continuous Media, Vol. 8 of Course of theoretical Physics, Pergamon Press, 2nd ed., 1984.

${ }^{11}$ Faux, I. D. and Pratt, M. J., Computational geometry for design and manufacture, Halsted Press (Wiley), 1979.

${ }^{12}$ Martino, W., Fernandez de la Mora, J., Yoshida, Y., Saito, G., and Wilkes, J., "Surface tension measurements of highly conducting ionic liquids," Green Chemistry, Vol. 8, 2006, pp. 390-397. 